IN S T I T U T O

DE

M E D I C I N A

T R O P I C A L

DE

SÃO PAULO

JOURNAL OF THE SÃO PAULO INSTITUTE OF TROPICAL MEDICINE

${ }^{1}$ Universidade Federal do Espírito Santo, Programa de Pós Graduação em Doenças Infecciosas, Vitória, Espírito Santo, Brazil

2Universidade Federal do Espírito Santo, Departamento de Ginecologia e Obstetrícia, Vitória, Espírito Santo, Brazil

${ }^{3}$ Universidade Federal do Espírito Santo, Programa de Residência Médica em Ginecologia e Obstetrícia, Vitória, Espírito Santo, Brazil

${ }^{4}$ UNIMED Vitória, Programa de Residência Médica em Ginecologia e Obstetrícia Vitória, Espírito Santo, Brazil.

Correspondence to: Helena Lucia Barroso dos Reis

Universidade Federal do Espírito Santo, Hospital Universitário Cassiano Antonio Moraes, Avenida Marechal Campos, 1468, Maruípe, CEP 29043-260 Vitória, ES, Brazil Tel: +55 27 3335-7180

E-mail: dr.hbarroso@gmail.com

Received: 20 June 2020

Accepted: 1 July 2020
http://doi.org/10.1590/S1678-9946202062049

\section{Severe coronavirus infection in pregnancy: challenging cases report}

\author{
Helena Lucia Barroso dos Reis ${ }^{(1)}$, Neide Aparecida Tosato Boldrini², \\ João Victor Jacomele Caldas ${ }^{3}$, Ana Paula Calazans da $\mathrm{Paz}^{4}$, Carolina Loyola \\ Prest Ferrugini' ${ }^{1}$, Angelica Espinosa Miranda ${ }^{1}$
}

\section{ABSTRACT}

There are few data on the impact of COVID-19 in pregnancy, however, analyzing these data is important to guide the clinical practice, covering the early prevention, detection, patients' isolation, epidemiological investigation, diagnosis and early treatment. This is a report of three cases of COVID-19 confirmed by real-time reverse transcription - polymerase chain reaction (RT-PCR) of nasopharyngeal secretions collected in swabs from pregnant women in the city of Vitoria, Espirito Santo State, Brazil. In the three cases, all the patients presented with fever, one had shortness of breath, one had diarrhea, two of them reported abdominal pain and two of them had cough. The three patients progressed with a severe clinical evolution of COVID-19. The permanence in the intensive care unit (ICU) was more than 10 days. Two of them recovered and one remained in the ICU with irreversible refractory shock, multiple organ failure and died. The mode of delivery was individualized and based on the obstetric indication and severity of the maternal infection, and the cesarean section was indicated in the two severe maternal COVID-19 cases that evolved favorably. These newborns were premature and tested negative for COVID-19 by RT-PCR.

KEYWORDS: COVID-19. SARS-CoV-2. Pregnancy. Infectious disease transmission. Severe acute respiratory syndrome.

\section{INTRODUCTION}

A new coronavirus known as the severe acute respiratory syndrome coronavirus 2 (SARS-CoV-2) was reported in 2019 and associated with pneumonia, causing a disease known as the coronavirus disease COVID-19. It is an emergent infectious disease that was first documented in Wuhan, China, and the World Health Organization (WHO) has declared it a global public health emergency and a pandemia ${ }^{1}$. Studies on the effects of COVID-19 during pregnancy are limited; however, owing to the high transmissibility of the virus and the possible development of severe acute respiratory symptoms, it is crucial that COVID-19 is investigated in the routine clinical practice of obstetrics.

Pregnant women represent an important challenge in the course of the pandemic. These patients need to attend to at least monthly medical consultations throughout the course of their prenatal care visits and for the delivery ${ }^{2}$. These appointments place them at a higher risk of contracting COVID-19 at the hospital environment or in outpatient clinics, although at this moment, the impact of COVID-19 on pregnancy and on newborn infants remains unclear. A Chinese study which tested amniotic fluid, cord blood, neonatal throat swabs, and breast milk samples from 
COVID-19-infected mothers found that all the samples tested negative for the virus, and another study determined that three placentas of pregnant women infected by COVID-19 tested negative for the virus ${ }^{3,4}$. However, the transmission rates from mothers to the newborns remain unknown. Assessment of clinical data to better understand the effects of COVID-19 in obstetrics will provide an opportunity to protect pregnant women, their offspring and the healthcare teams involved in their care.

This study reports three cases of COVID-19 comprising pregnant women with severe respiratory failure and evaluates the clinical management of COVID-19 infection in obstetric clinics in Vitoria, Espirito Santo State, Brazil.

\section{CASE REPORTS}

\section{Case 1}

A 28-year-old pregnant woman who worked as a nursing assistant, was a primigravida with 31 weeks of gestation age who sought the emergency unit with fever and hyperemic lesions in the right lower limb, suggestive of a bacterial skin infection. The patient did not smoke or report a prior history of asthma, tuberculosis or pneumonia. Treatment was initiated with intravenous ceftriaxone $(2 \mathrm{~g})$ associated with $40 \mathrm{IU}$ per day of heparin, though after two days, the patient began to exhibit acute dyspnea. The room oxygen saturation was found to be $95 \%$, and symptoms were accompanied by tachypnea and hypotension. Fifteen liters of oxygen per minute were provided by mask, and the patient was transferred to the intensive care unit (ICU) of another public institution. She was admitted in critical conditions requiring urgent intubation and mechanical ventilation. The lactate dehydrogenase $(\mathrm{LDH})$ was $268 \mathrm{U} / \mathrm{L}$, D-dimer less than $300 \mathrm{ng} / \mathrm{mL}$ (normal values below $500 \mathrm{ng} / \mathrm{mL}$ ) and 3,900 white blood cells (WBC). Computed tomography (CT) scans revealed bilateral patchy ground glass opacities, and the patient was considered as a suspicious case of SARS-CoV-2 infection. Considering the CT findings and the severe evolution in the third trimester of gestation, she was medicated with $500 \mathrm{mg}$ azithromycin per day, $75 \mathrm{mg}$ oseltamivir phosphate twice a day and $400 \mathrm{mg}$ hydroxychloroquine twice a day for five days, in addition to the ventilation support. Pulmonary embolism was excluded by using specific diagnostic tests.

She tested negative for coronavirus by the real-time reverse transcription polymerase chain reaction (RT-PCR) testing nasopharyngeal secretions swabs during the initial hospitalization. Owing to the severe clinical conditions, a cesarean section (C-section) was performed. A healthy 31-week gestational age infant, weighting 2,380 g, was delivered and transferred to the neonatal care unit because of the prematurity, the Apgar score was 8 on the $1^{\text {st }}$ minute of life and 9 on the $5^{\text {th }}$ minute and the preterm newborn was adequate for gestation age. The puerperal woman remained hospitalized for 18 days in the intensive care unit (ICU) due to her respiratory conditions, receiving supportive care. The patient tested positive for COVID-19 by RT-PCR on the $12^{\text {th }}$ day after hospitalization. The newborn tested negative for COVID-19 by RT-PCR and was released after 5 days. The puerperal woman evolved favorably and was discharged without sequelae after 23 days.

\section{Case 2}

A 34-year-old puerperal woman who was a housewife in her second pregnancy, with one previous delivery, sought the emergency unit on the fifth day after delivery owing to an acute respiratory insufficiency. She did not report abnormalities during the antenatal care or during hospitalization for delivery. Her medical record showed that she gave birth by C-section at 40 weeks of gestational age and gave birth to a female newborn, weighing $3,875 \mathrm{~g}$, with an Apgar score of 8 and 9 in the $1^{\text {st }}$ and $5^{\text {th }}$ minutes, respectively. The mother reported severe dry cough and acute dyspnea associated with lower back pain and mild fever occurring 24 hours before the hospitalization. The chest CT scan was suggestive of viral pneumonia with an acute inflammatory feature compromising 25 to $40 \%$ of the pulmonary parenchyma, but the findings were not suggestive of COVID-19. The angiotomograpy of the thorax was negative for pulmonary embolism and the oxygen saturation was $91 \%$. The LDH was $328 \mathrm{U} / \mathrm{L}$, D-dimer less than $300 \mathrm{ng} / \mathrm{mL}$ and the initial leukogram showed 5,000 WBC.

The patient was medicated with ceftriaxone ( $2 \mathrm{~g} /$ day), azithromycin (500 mg/day), oseltamivir (75 mg twice a day), and enoxaparin (initially with $40 \mathrm{mg}$ daily, with increments up to $80 \mathrm{mg} /$ day). The RT-PCR for SARS-CoV-2 was positive three days after the delivery, and hydroxychloroquine (400 mg twice a day) was administered initially, followed by 400 $\mathrm{mg}$ daily for five subsequent days along with meropenem (500 $\mathrm{mg}$ three times a day) and vancomycin $(1 \mathrm{~g}$ twice a day). Ceftriaxone was discontinued.

Afterwards, the patient's respiratory condition worsened, she remained in the ICU in critical conditions with a persistent high fever that last 13 days, receiving supportive care covering oxygenation, ventilation, and vasoactive drugs. The patient no longer needed the ICU hospitalization following this period and was transferred to the rehabilitation ward. Her husband and the newborn tested negative for COVID-19. 


\section{Case 3}

A 25-year-old pregnant woman, who was a housewife in her third pregnancy and two previous deliveries, was referred to the hospital at 28 weeks and six days of gestational age with upper respiratory symptoms, fever, myalgia, and diarrhea for five days. Thereafter, the patient's clinical condition worsened to a non-productive cough and intense fatigue. She did not report comorbidities, but her husband presented with fever in the previous 24 hours and reported contact with two coworkers that had positive tests for COVID-19. She was transferred to the ICU. Corticosteroid therapy with $2 \mathrm{~g}$ hydrocortisone was initiated to accelerate the fetal lung maturation along with oseltamivir, azithromycin, ceftriaxone, and enoxaparin in standardized doses. The patient also needed mechanical ventilation. D-dimer levels were normal (308 ng/mL), and pulmonary embolism was excluded using specific diagnostic tests. The oxygen saturation was $94 \%$ and the initial leukogram showed 4,000 WBC and $11 \%$ of lymphocytes.

The COVID-19 serological tests resulted negative twice, while the RT-PCR on nasopharyngeal swab secretions was positive for SARS-CoV-2. Hydroxychloroquine was initiated (400 mg twice a day), followed by $400 \mathrm{mg}$ daily for five subsequent days. A C-section was performed owing to the critical conditions of the mother, as well as severe oligohydramnios.

A female infant weighing $1,255 \mathrm{~g}$, adequate for gestational age was delivered. The Apgar score was 6 on the $1^{\text {st }}$ minute and 7 on the $5^{\text {th }}$ minute. The newborn tested negative for COVID-19 by RT-PCR, but was referred to the neonatal ICU because of the extreme prematurity, where she underwent a systemic antibiotic therapy owing to a right hemi thorax atelectasis. The neonate was released after 30 days in good conditions.

The mother remained in the ICU for 22 days, where she was treated with meropenem $(500 \mathrm{mg}$ three times a day) associated with vancomycin (1 g twice a day) and polymyxin-B, requiring intubation and mechanical ventilation. The $\mathrm{LDH}$ result went from $226 \mathrm{U} / \mathrm{L}$ to $524 \mathrm{U} / \mathrm{L}$ during the hospitalization. . Despite the ICU stay and a continuous epinephrine drip, the patient's blood pressure decreased to $60 / 40 \mathrm{mmHg}$ and her condition evolved to irreversible refractory shock, multiple organ failure and death. The blood cultures were all negative.

\section{DISCUSSION}

There is not yet unequivocal scientific evidence of mother-to-child transmission (MTCT) of COVID-19; whether a cesarean section could prevent transmission, whether vaginal delivery is free of additional risks, or whether the time of delivery should be anticipated ${ }^{2,3}$.

Current recommendations are that cases should be individualized according to the instruction of the obstetrician and the degree of severity of the maternal conditions ${ }^{2}$. However, it is consensual that infection by the SARS-CoV-2 virus during pregnancy may increase the risk of maternal and fetal health deterioration because COVID-19 is an infectious disease that can evolve to severe pneumonia and admissions to the ICU, potentially resulting in the anticipation of deliveries before the pregnancy full term ${ }^{4}$. This situation occurred in two of the three cases reported, in all the cases, the patients presented with fever and the patients' clinical conditions worsen quickly.

The patients' ICU hospitalization were all more than 10 days. Two of them recovered, and one remained in the ICU and developed refractory shock, progressing to death.

The newborns were premature in two of the three cases due to the need to perform the C-section, but none of the newborns presented symptoms of infection, and all three tested negative for COVID-19 using RT-PCR that tested nasopharyngeal secretions. Two of the newborns were only admitted to the ICU because of their prematurity. Table 1 summarizes the findings in the three cases.

In Brazil, there are other suggested cases of maternal mortality due to COVID- $19^{5}$. It can also be noted that the conditions of the women infected with SARS-CoV-2 during pregnancy progressed within a short period from the infection transmission and incubation period. Case 1 was admitted with a suggested bacterial skin infection, and her condition progressed to pneumonia in a few days. A previous study in China described $12.6 \%$ cases of COVID-19 presymptomatic patients; they have also reported a mean interval of 3.96 days between presymptoms and the diagnosis ${ }^{6}$. It is important to be attentive and perform a good anamnesis and physical examination to identify these situations.

According to another Chinese study, the characteristics of pneumonia in pregnant women with COVID-19 do not differ from pneumonia in non-pregnant women. In a small group of nine infected pregnant women, there was no evidence of vertical transmission of COVID-19 in those who acquired the virus and developed pneumonia at the end of the pregnancy. All the patients underwent a cesarean section and all the newborns were tested for the virus and had negative results ${ }^{3}$. Another study including 43 confirmed COVID-19 pregnant women in New York city reported a $4.7 \%$ rate of critical illness; these data were similar to the ones described for non-pregnant adults with COVID-19 infections ${ }^{7}$. Similar findings were also observed in a study 
Table 1 - Summary of data from the three pregnant women diagnosed with COVID-19 and their initial diagnoses.

\begin{tabular}{|c|c|c|c|}
\hline Variables & Patient 1 & Patient 2 & Patient 3 \\
\hline Age (years) & 28 & 34 & 25 \\
\hline Gravida (parity) & $1(0)$ & $1(1)$ & $3(2)$ \\
\hline Gestational age at admission in weeks & 31 & 1 day after delivery & 28 and 6 days \\
\hline Fever (days) & Yes (3) & Yes (4) & Yes (14) \\
\hline Cough & No & Yes & Yes \\
\hline Shortness of breath & No & Yes & Yes \\
\hline Diarrhoea & No & No & Yes \\
\hline Abdominal pain & Yes & No & Yes \\
\hline Previous comorbidities & No & No & No \\
\hline Delivery mode* & C-section & C-section & C-section \\
\hline Birth outcome & Preterm & Term & Preterm \\
\hline Apgar Score on the $1^{\text {st }}$ and $5^{\text {th }}$ minutes & $8: 9$ & $8: 9$ & $6: 7$ \\
\hline Fetal Growth & AGA & AGA & AGA \\
\hline Infants' COVID-19 RT-PCR & Negative & Negative & Negative \\
\hline Pregnancy complications & No & No & No \\
\hline Initial leukogram & $3,900 / \mathrm{mm}^{3}$ & $5,000 / \mathrm{mm}^{3}$ & $\begin{array}{c}4,000 / \mathrm{mm}^{3} \\
\text { Lymphocytes } 11 \%\end{array}$ \\
\hline $\mathrm{SPO}_{2}-\mathrm{ARDS}$ & $95-70 \%$ & $91-60 \%$ & $94-60 \%$ \\
\hline Serial C-reactive protein $(\mathrm{mg} / \mathrm{L})$ : & $8 \rightarrow 36.5$ & $70 \rightarrow 78.6$ & $55.1 \rightarrow 163.4$ \\
\hline $\mathrm{LDH}(\mathrm{U} / \mathrm{L})$ & 268 & 328 & 540 \\
\hline D-dimer (ng/mL) & $<300$ & $<300$ & 308 \\
\hline Serial blood cultures & NA & NA & Negative \\
\hline
\end{tabular}

${ }^{*} \mathrm{C}$-section was indicated because of the severity of the infection in two cases. AGA = Adequate for gestational age; Elevated C-reactive protein (mg/L) was considered $\geq 10 \mathrm{mg} / \mathrm{L}$; Normal D-dimer value up to $500 \mathrm{ng} / \mathrm{mL} ; \mathrm{O}_{2}$ values are as reported at the time of diagnosis of ARDS and intubation; ARDS = Acute respiratory distress syndrome; RT-PCR = Real-time reverse transcription polymerase chain reaction; $N A=$ Not available; $\mathrm{LDH}=$ Lactate dehydrogenase.

of pregnant Chinese women infected with COVID-19 in which there were no maternal deaths or confirmed cases of intrauterine transmission of the infection to the fetuses ${ }^{8,9}$. In the three cases examined here, all the patients initially presented with fever; one had shortness of breath, one had diarrhea, two reported abdominal pain, and two had cough. All three patients' clinical conditions progressed rapidly.

There are still gaps regarding the MTCT and newborn infection; a case report of a pregnant woman with coronavirus infection and a newborn infected with SARS-CoV-2 36 hours after delivery was identified; however, confirmation of MTCT was not established ${ }^{10}$. A possible case of MTCT was also described elsewhere, but the newborn's RT-PCR result was negative ${ }^{11}$. Children with COVID-19 seem not to be frequently recognized, and most of those identified had only mild symptoms ${ }^{12}$.

In addition, a morphological placental study of a series of three neonatal cases of mothers infected with COVID-19 found no changes related to the infection ${ }^{4}$.
Unfortunately, amniotic fluid and placenta were not tested by RT-PCR in our cases. In the patients examined here, two of the newborns were premature because of the urgency of the C-section, but they did not present symptoms of the SARS-CoV-2 infection, indicating there was no MTCT. It is also uncertain whether our institution's empiric administration of hydroxychloroquine enabled the patients' recovery (cases 1 and 2). Currently, there is no official recommendation of chloroquine or hydroxychloroquine for COVID-19 treatment. The use of these drugs in pregnant women should only be considered if their benefits outweight the adverse effects ${ }^{13}$.

An interesting finding in this study was that case 1 was a health professional; this highlights the extra concern about the security measures regarding the protection of front-line workers from contamination. In Brazil, the only study on COVID-19 during pregnancy to date has been a review of initial data on pregnancy and the implications for assisted reproductive treatments; this study recommended 
postponing pregnancies ${ }^{14}$. As COVID-19 is a new challenge for health professionals and its impact on mothers and children is unknown, new studies are needed to understand the impact on pregnant women to generate evidence for improving its clinical management, treatment, and prevention.

\section{CONCLUSION}

There is still no clinical evidence regarding the timing of delivery, vertical transmission, or the safety of vaginal delivery in pregnant women with COVID-19. The mode of delivery must be individualized, based on the obstetric indication, the severity of the maternal infection, and cesarean sections should be indicated in severe cases. To our knowledge, this is one of the first published reports on the death of a Brazilian pregnant women with confirmed COVID-19 infection.

\section{CONFLICT OF INTERESTS}

The authors have no conflict of interests to declare.

\section{ETHICS}

The women had not identified their identity compromised. The ethical review board of the university hospital approved the study.

\section{FUNDING}

This research did not receive any specific grant from funding agencies in the public, commercial, or not-forprofit sectors.

\section{REFERENCES}

1. Zhu N, Zhang D, Wang W, Li X, Yang B, Song J, et al. A novel coronavirus from patients with pneumonia in China, 2019. N Engl J Med. 2020;382:727-33.

2. Chen D, Yang H, Cao Y, Cheng W, Duan T, Fan C, et al. Expert consensus for managing pregnant women and neonates born to mothers with suspected or confirmed novel coronavirus (COVID-19) infection. Int J Gynaecol Obstet. 2020;149:130-6.
3. Chen H, Guo J, Wang C, Luo F, Yu X, Zhang W, et al. Clinical characteristics and intrauterine vertical transmission potential of COVID-19 infection in nine pregnant women: a retrospective review of medical records. Lancet. 2020;395:809-15.

4. Chen S, Huang B, Luo DJ, Li X, Yang F, Zhao Y, et al. Pregnant women with new coronavirus infection: a clinical characteristics and placental pathological analysis of three cases. Zhonghua Bing Li Xue Za Zhi. 2020;49:418-23.

5. Amorim MM, Soligo Takemoto ML, Fonseca EB. Maternal deaths with coronavirus disease 2019: a different outcome from lowto middle-resource countries? Am J Obstet Gynecol. 2020 In Press.

6. Du Z, Xu X, Wu Y, Wang L, Cowling BJ, Meyers LA. Serial interval of COVID-19 among publicly reported confirmed cases. Emerg Infect Dis. 2020;26:1341-43.

7. Breslin N, Baptiste C, Gyamfi-Bannerman C, Miller R, Martinez R, Bernstein K, et al. COVID-19 infection among asymptomatic and symptomatic pregnant women: Two weeks of confirmed presentations to an affiliated pair of New York City hospitals. Am J Obstet Gynecol MFM. 2020;2:100118.

8. Schwartz DA. An analysis of 38 Pregnant women with COVID-19, their newborn infants, and maternal-fetal transmission of SARS-CoV-2: maternal coronavirus infections and pregnancy outcomes. Arch Pathol Lab Med. 2020 In Press.

9. Zhu H, Wang L, Fang C, Peng S, Zhang L, Chang G, et al. Clinical analysis of 10 neonates born to mothers with 2019-nCoV pneumonia. Transl Pediatr. 2020;9:51-60.

10. Wang S, Guo L, Chen L, Liu W, Cao Y, Zhang J, Feng L. A case report of neonatal COVID-19 infection in China. Clin Infect Dis. 2020:ciaa225 In Press.

11. Dong L, Tian J, He S, Zhu C, Wang J, Liu C, Yang J. Possible vertical transmission of SARS-CoV-2 from an infected mother to her newborn. JAMA. 2020;323:1846-8.

12. Shen KL, Yang YH. Diagnosis and treatment of 2019 novel coronavirus infection in children: a pressing issue. World $\mathrm{J}$ Pediatr. 2020;16:219-21.

13. Lacroix I, Bénévent J, Damase-Michel C. Chloroquine and hydroxychloroquine during pregnancy: what do we know? Therapie. 2020 In Press.

14. Monteleone PA, Nakano M, Lazar V, Gomes AP, Martin H, Bonetti TC. A review of initial data on pregnancy during the COVID-19 outbreak: implications for assisted reproductive treatments. JBRA Assist Reprod. 2020;24:219-25. 